\title{
Cerámicas dentales: clasificación y criterios de selección
}

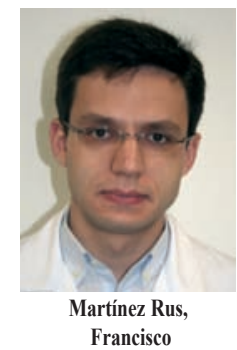

\author{
Dental ceramics: Classification and selection criteria
}

\section{Marlinez Aus, Francisco* Pradíes Ramiro, Guillermo** Suárez García, Mả Ješ́ls** Rivera Gómez, Begoña ***}

\footnotetext{
* Profesor Asociado. Departamento de Prótesis Bucofacial. Facultad de Odontología. Universidad Complutense de Madrid.

** Profesor/a Titular. Departamento de Prótesis Bucofacial. Facultad de Odontología. Universidad Complutense de Madrid.

*** Profesora Ayudante. Departamento de Ciencias de la Salud III. Facultad de Ciencias de la Salud. Universidad Rey Juan Carlos.
}

\section{Correspondencia}

Francisco Martínez Rus

Departamento de Prótesis Bucofa-

cial.

Facultad de Odontología.

Universidad Complutense de

Madrid.

Plaza Ramón y Cajal, s/n. 28040 Madrid (España)

E-mail: framarti@odon.ucm.es
Resumen : Hoy en día, hablar de restauraciones estéticas implica hablar de cerámica sin metal. Han sido tan importantes y revolucionarios los cambios y aportaciones en este campo en los últimos años que en la actualidad existen multitud de sistemas cerámicos. Todos ellos buscan el equilibrio entre los factores estéticos, biológicos, mecánicos y funcionales. Sin embargo, existen diferencias considerables entre ellos. Por lo tanto, para seleccionar la cerámica más adecuada en cada caso, es necesario conocer las principales características de estos materiales y de sus técnicas de confección. Esta elección no debe ser delegada al técnico de laboratorio, sino que debe ser responsabilidad del odontoestomatólogo porque él es quien conoce y controla las variables que condicionan el éxito de la restauración a largo plazo. En este artículo, se revisan los principales sistemas cerámicos disponibles actualmente y se analiza su comportamiento clínico. Por último, se exponen unas pautas para orientar al profesional en la toma de decisiónes.

Palabras clave: Cerámica, Composición química, Técnica de confección, Resistencia a la fractura, Ajuste marginal, Estética, Supervivencia clínica.

Abstract: At the present time, to speak about aesthetic restorations implies speaking about alloy free ceramics. This field has experienced important changes and revolutionary contributions. This has led to the introduction of a multitude of all-ceramic systems. All of these quest for a balance between the aesthetic, biological, mechanical and functional factors. However, considerable differences exist among them. Therefore, to select the most suitable ceramic in every case, it is necessary to know the main features of these materials and the laboratory procedures. Porcelain selection should not be left up to the laboratory technician. Material selection should be the responsibility of the clinician because he knows and controls the variables that determine the long-term success of the restoration. This article reviews the all-ceramic systems now available and its clinical performance. Lastly, decision making guidelines for the clinician are detailed.

Key words: Ceramics, chemical composition, laboratory procedure, fracture strength, marginal fit, aesthetics and clinical survival.

\section{BIBLID [1138-123X (2007)12:4; octubre-diciembre 209-316]}

Martínez Rus F, Pradíes Ramiro G, Suárez García MJ, Rivera Gómez B. Cerámicas dentales: clasificación y criterios de selección. RCOE 2007;12(4):253263. 


\section{Introducción}

Las restauraciones ceramometálicas son la base del modelo actual de prótesis fija. Pero, a pesar de su contrastado éxito, no han cesado los esfuerzos por lograr sistemas totalmente cerámicos debido a la necesidad de encontrar prótesis más estéticas y más biocompatibles. La estética es un concepto subjetivo, sometido a grandes cambios según el medio socio-cultural que se trate. Pero no cabe duda de que en el entorno en que nos movemos hablar de restauraciones estéticas en el momento actual, implica hablar de cerámica sin metal. Además, las porcelanas son más inertes que los metales. Sabemos que las aleaciones pueden verter iones nocivos al medio oral al sufrir corrosión, hecho que no ocurre en las cerámicas debido a su baja reactividad química.

A pesar de que a principios del siglo XX, ya se realizaban coronas «jackets» de porcelana, el gran desarrollo de las restauraciones completamente cerámicas se ha producido en las últimas dos décadas debido a la gran profusión de innovaciones tecnológicas y materiales. Han sido tan importantes y revolucionarios los cambios y aportaciones en este campo en los últimos años que en la actualidad existen multitud de sistemas cerámicos. Todos ellos buscan el equilibrio entre los factores estéticos, biológicos, mecánicos y funcionales. De manera que la cerámica sin metal hoy en día no sólo se usa para confeccionar restauraciones unitarias del sector anterior, como clásicamente se indicaba, sino que también se aplica a los sectores posteriores y a la elaboración

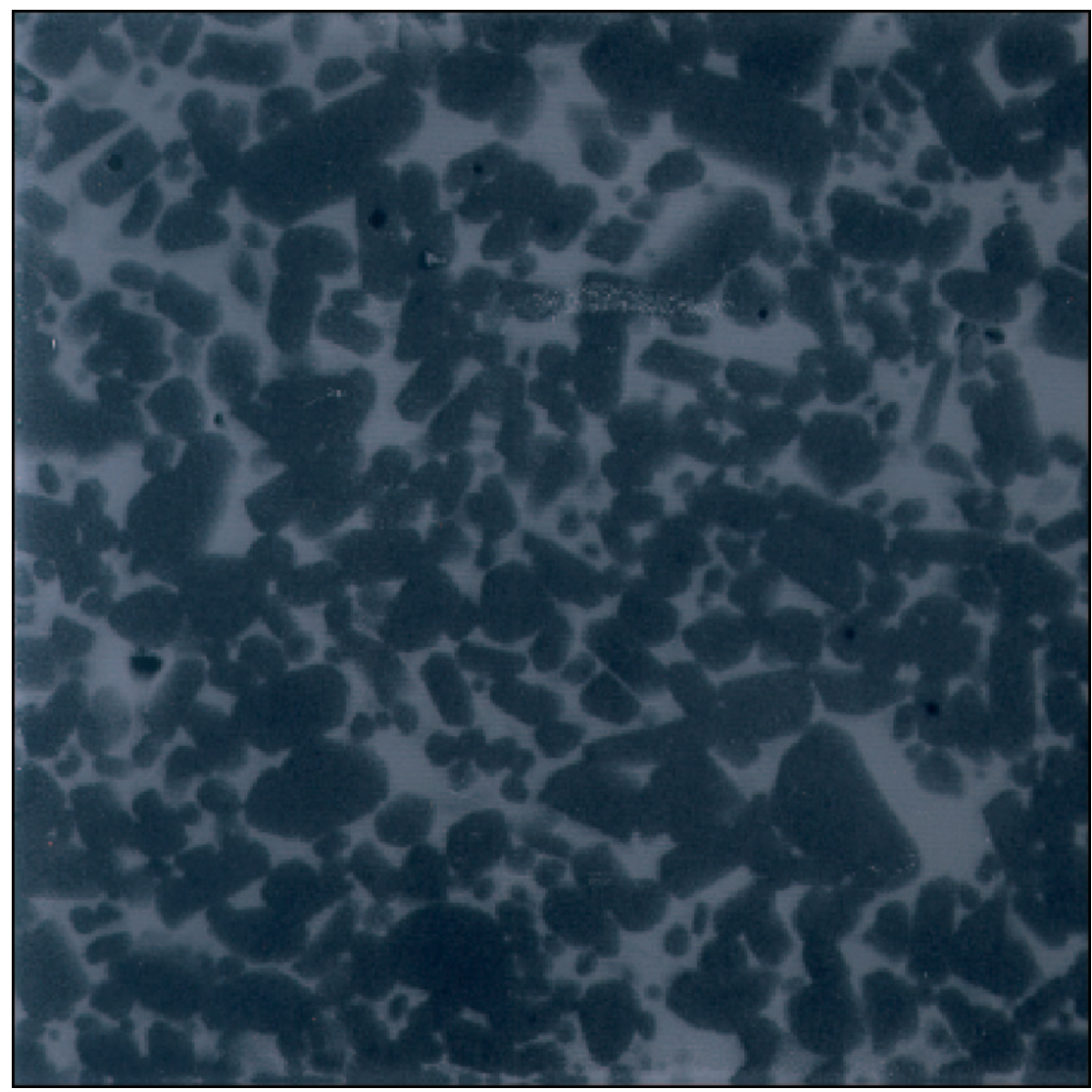

Figura 1. Microestructura de la cerámica In Ceram ${ }^{\circledR}$ Alumina (Vita).

de puentes. El objetivo de esta publicación es ofrecer una revisión ordenada de un tema en el que todavía existe una gran confusión debido a la enorme heterogeneidad de estos materiales.

\section{Clasificación de los sistemas totalmente cerámicos}

A pesar de que las clasificaciones son totalmente artificiales, siempre nos ayudan porque permiten organizar mejor los conocimientos sobre una determinada materia. Por ello, vamos a agrupar los sistemas totalmente cerámicos en función de dos criterios: composición química y técnica de confección ${ }^{1-3}$

\section{Clasificación por la com- posición química}

Antes de entrar en materia conviene recordar algunos conceptos básicos sobre la composición química de las cerámicas. Se consideran materiales cerámicos aquellos productos de naturaleza inorgánica, formados mayoritariamente por elementos no metálicos, que se obtienen por la acción del calor y cuya estructura final es parcial o totalmente cristalina. La gran mayoría de las cerámicas dentales, salvo excepciones que comentaremos, tienen una 
estructura mixta, es decir, son materiales compuestos formados por una matriz vítrea (cuyos átomos están desordenados) en la que se encuentran inmersas partículas más o menos grandes de minerales cristalizados (cuyos átomos si que están dispuestos uniformemente) (fig. 1). Es importante señalar que la fase vítrea es la responsable de la estética de la porcelana mientras que la fase cristalina es la responsable de la resistencia. Por lo tanto, la microestructura de la cerámica tiene una gran importancia clínica ya que el comportamiento estético y mecánico de un sistema depende directamente de su composición. Por ello, conviene recordar los cambios estructurales que se han producido en las porcelanas a lo largo de la historia hasta llegar a las actuales cerámicas. Químicamente, las porcelanas dentales se pueden agrupar en tres grandes familias: feldespáticas, aluminosas y circoniosas.

\section{Cerámicas feldespáticas}

Las primeras porcelanas de uso dental tenían la misma composición que las porcelanas utilizadas en la elaboración de piezas artísticas. Contenían exclusivamente los tres elementos básicos de la cerámica: feldespato, cuarzo y caolín. Con el paso del tiempo, la composición de estas porcelanas se fue modificando hasta llegar a las actuales cerámicas feldespáticas, que constan de un magma de feldespato en el que están dispersas partículas de cuarzo y, en mucha menor medida, caolín. El feldespato, al descomponerse en vidrio, es el responsable de la translucidez de la porcelana. El cuarzo constituye la fase cristalina. El caolín confiere plasticidad y facilita el manejo de la cerámica cuando todavía no esta cocida. Además, para disminuir la temperatura de sinterización de la mezcla siempre se incorporan "fundentes». Conjuntamente, se añaden pigmentos para obtener distintas tonalidades. Al tratarse básicamente de vidrios poseen unas excelentes propiedades ópticas que nos permiten conseguir unos buenos resultados estéticos; pero al mismo tiempo son frágiles y, por lo tanto, no se pueden usar en prótesis fija si no se «apoyan» sobre una estructura. Por este motivo, estas porcelanas se utilizan principalmente para el recubrimiento de estructuras metálicas o cerámicas.

Como ya señalamos, debido a la demanda de una mayor estética en las restauraciones, se fue modificando la composición de las cerámicas hasta encontrar nuevos materiales que tuvieran una tenacidad adecuada para confeccionar restauraciones totalmente cerámicas. En este contexto surgieron las porcelanas feldespáticas de alta resistencia. Éstas tienen una composición muy similar a la anteriormente descrita. Poseen un alto contenido de feldespatos pero se caracterizan porque incorporan a la masa cerámica determinados elementos que aumentan su resistencia mecánica (100-300 $\mathrm{MPa})$. Entre ellas encontramos:

- Optec-HSP® (Jeneric), Fortress ${ }^{\circledR}$ (Myron Int), Finesse ${ }^{\circledR}$ AllCeramic (Dentsply) e IPS Empress ${ }^{\circledR}$ । (Ivoclar): Deben su resistencia a una dispersión de microcristales de leucita, repartidos de forma uniforme en la matriz vítrea. La leucita refuerza la cerámica porque sus partículas al enfriarse sufren una reducción volumétrica porcentual mayor que el vidrio circundante.
Esta diferencia de volumen entre los cristales y la masa amorfa genera unas tensiones residuales que son las responsables de contrarrestar la propagación de grietas.

- IPS Empress $®$ II (Ivoclar): Este sistema consta de una cerámica feldespática reforzada con disilicato de litio y ortofosfato de litio. La presencia de estos cristales mejora la resistencia pero también aumenta la opacidad de la masa cerámica. Por ello, con este material solamente podemos realizar la estructura interna de la restauración. Para conseguir un buen resultado estético, es necesario recubrir este núcleo con una porcelana feldespática convencional.

- IPS e.max® Press/CAD (Ivoclar): Estas nuevas cerámicas feldespáticas están reforzadas solamente con cristales de disilicato de litio. No obstante, ofrecen una resistencia a la fractura mayor que Empress ${ }^{\circledR}$ ॥ debido a una mayor homogeneidad de la fase cristalina. Al igual que en el sistema anterior, sobre estas cerámicas se aplica una porcelana feldespática convencional para realizar el recubrimiento estético mediante la técnica de capas.

\section{Cerámicas aluminosas}

En 1965, McLean y Hughes abrieron una nueva vía de investigación en el mundo de las cerámicas sin metal. Estos autores incorporaron a la porcelana feldespática cantidades importantes de óxido de aluminio reduciendo la proporción de cuarzo. El resultado fue un material con una microestructura mixta en la que la alúmina, al tener una temperatura de fusión elevada, permanecía en suspensión en la matriz. 
Estos cristales mejoraban extraordinariamente las propiedades mecánicas de la cerámica. Esta mejora en la tenacidad de la porcelana animó a realizar coronas totalmente cerámicas.

sin embargo, pronto observaron que este incremento de óxido de aluminio provocaba en la porcelana una reducción importante de la translucidez, que obligaba a realizar tallados agresivos para alcanzar una buena estética. Cuando la proporción de alúmina supera el $50 \%$ se produce un aumento significativo de la opacidad. Por este motivo, en la actualidad las cerámicas de alto contenido en óxido de aluminio se reservan únicamente para la confección de estructuras internas, siendo necesario recubrirlas con porcelanas de menor cantidad de alúmina para lograr un buen mimetismo con el diente natural. Los sistemas más representativos son:

- In-Ceram® Alumina (Vita): Para fabricar las estructuras de coronas y puentes cortos utiliza una cerámica compuesta en un $99 \%$ por óxido de aluminio, lógicamente sin fase vítrea. Sin embargo, como en la sinterización no se alcanza la máxima densidad, el material resultante se infiltra con un vidrio que difunde a través de los cristales de alúmina por acción capilar para eliminar la porosidad residual. Esto permite obtener un núcleo cerámico más resistente a la flexión.

- In-Ceram® Spinell (Vita): Incorpora magnesio a la fórmula anterior. El óxido de magnesio (28\%) junto con el óxido de aluminio (72\%) forma un compuesto denominado espinela (MgAl204). La principal ventaja de este sistema es su excelente estética debido a que estos crista-

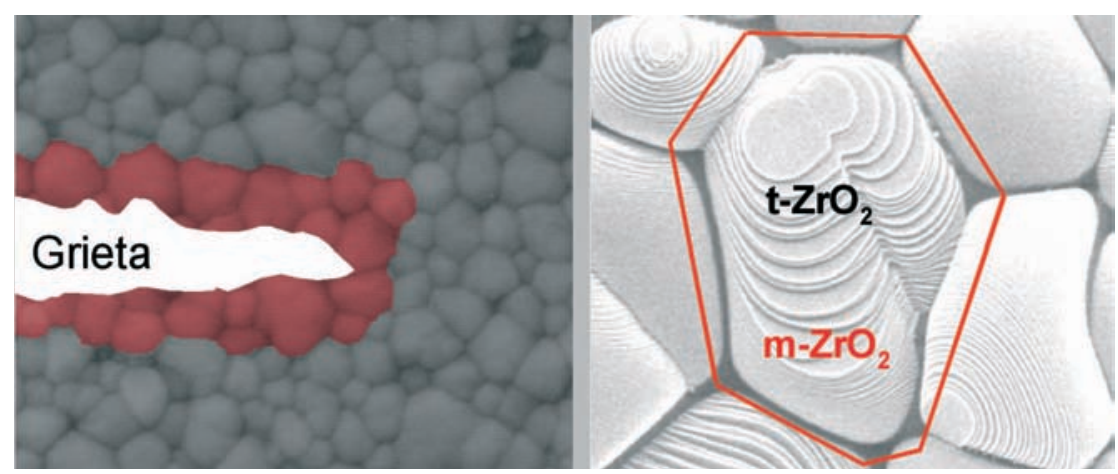

Figura 2. Transformación de fase cristalina en la circonia.

les por sus características ópticas isotrópicas son más translúcidos que los de alúmina. No obstante, estas cofias presentan un 25\% menos de resistencia a la fractura que las anteriores, a pesar de que también se les infiltra con vidrio tras su sinterización. Por ello, esta indicado solamente para elaborar núcleos de coronas en dientes vitales anteriores.

- In-Ceram ${ }^{\circledR}$ Zirconia (Vita): Estas restauraciones se caracterizan por una elevada resistencia, ya que sus estructuras están confeccionadas con un material compuesto de alúmina $(67 \%)$ reforzada con circonia (33\%) e infiltrado posteriormente con vidrio. El oxido de circonio aumenta significativamente la tenacidad y la tensión umbral de la cerámica aluminosa hasta el punto de permitir su uso en puentes posteriores.

- Procera ${ }^{\circledR}$ AllCeram (Nobel Biocare): Este sistema emplea una alúmina de elevada densidad y pureza (>99,5\%). Sus cofias se fabrican mediante un proceso industrial de prensado isostático en frío y sinterización final a $1550^{\circ} \mathrm{C}$. Con esta técnica, el material se compacta hasta su densidad teórica, adquiriendo una microestructura completamente cristalina. El resultado es una cerámica con una alta resistencia mecánica porque al desaparecer el espacio residual entre los cristales se reduce la aparición de fisuras.

\section{Cerámicas circoniosas}

Este grupo es el más novedoso. Estas cerámicas de última generación están compuestas por óxido de circonio altamente sinterizado (95\%), estabilizado parcialmente con óxido de itrio (5\%). El óxido de circonio $\left(\mathrm{ZrO}_{2}\right)$ también se conoce químicamente con el nombre de circonia o circona. La principal característica de este material es su elevada tenacidad debido a que su microestructura es totalmente cristalina y además posee un mecanismo de refuerzo denominado "transformación resistente». Este fenómeno descubierto por Garvie \& cols. en 1975 consiste en que la circonia parcialmente estabilizada ante una zona de alto estrés mecánico como es la punta de una grieta sufre una transformación de fase cristalina, pasa de forma tetragonal a monoclínica, adquiriendo un volumen mayor (fig. 2). De este modo, 
se aumenta localmente la resistencia y se evita la propagación de la fractura. Esta propiedad le confiere a estas cerámicas una resistencia a la flexión entre 1000 y 1500 MPa, superando con una amplio margen al resto de porcelanas. Por ello, a la circonia se le considera el "acero cerámico». Estas excelentes características físicas han convertido a estos sistemas en los candidatos idóneos para elaborar prótesis cerámicas en zonas de alto compromiso mecánico. A este grupo pertenecen las cerámicas dentales de última generación: DC-Zircon ${ }^{\circledR}$ (DCS), Cercon ${ }^{\circledR}$ (Dentsply), In-Ceram ${ }^{\circledR}$ YZ (Vita), Procera ${ }^{\circledR}$ Zirconia (Nobel Biocare), Lava ${ }^{\circledR}$ (3M Espe), IPS e.max $®$ ZirCAD (Ivoclar), etc. Al igual que las aluminosas de alta resistencia, estas cerámicas son muy opacas (no tienen fase vítrea) y por ello se emplean únicamente para fabricar el núcleo de la restauración, es decir, deben recubrirse con porcelanas convencionales para lograr una buena estética.

El nuevo reto de la investigación es aumentar la fiabilidad de las actuales cerámicas monofásicas aluminosas y circoniosas. Recientemente, se ha demostrado que la circonia tetragonal metaestable en pequeñas proporciones $(10-15 \%)$ refuerza la alúmina de forma significativa4. Estos "composites" altamente sinterizados alcanzan unos valores de tenacidad y de tensión umbral mayores que los conseguidos por la alúmina y la circonia de forma individual. Además, tienen una adecuada dureza y una gran estabilidad química. Así pues, estos biomateriales de alúmina-circonia se presentan como una alternativa a tener en cuenta en el futuro para la confección de restauraciones cerámicas.

\section{Clasificación por la técnica de confección}

La clasificación de las cerámicas analizando exclusivamente la forma de confección en el laboratorio es bastante útil y representativa. Siguiendo este criterio, los sistemas cerámicos se pueden clasificar en tres grupos: condensación sobre muñón refractario, sustitución a la cera perdida y tecnología asistida por ordenador.

\section{Condensación sobre muñón refrac- tario}

Esta técnica se basa en la obtención de un segundo modelo de trabajo, duplicado del modelo primario de escayola, mediante un material refractario que no sufre variaciones dimensionales al someterlo a las temperaturas que requiere la cocción de la cerámica. La porcelana se aplica directamente sobre estos troqueles termoresistentes. Una vez sinterizada, se procede a la eliminación del muñón y a la colocación de la prótesis en el modelo primario para las correcciones finales. Son varios los sistemas que utilizan este procedimiento: Optec-HSP ${ }^{\circledR}$ (Jeneric), Fortress ${ }^{\circledR}$ (Myron Int), InCeram ${ }^{\circledR}$ Spinell (Vita), etc.

\section{Sustitución a la cera pérdida}

Este método está basado en el tradicional modelado de un patrón de cera que posteriormente se transforma mediante inyección en una estructura cerámica, tal y como clásicamente se efectúa con el metal. Inicialmente se encera el patrón que puede representar la cofia interna o la restauración completa. Una vez realizado el patrón, se reviste en un cilindro y se procede a calcinar la cera. A continuación, se calienta la cerámica (que se presenta en forma de pastillas) hasta su punto de fusión. El paso del material hacia el interior del cilindro se realiza por inyección, en donde un pistón va empujando la cerámica fluida hasta el molde. Los sistemas más representativos son IPS Empress ${ }^{\circledR}$ y e.max ${ }^{\circledR}$ Press (Ivoclar). Diversos estudios han demostrado que este procedimiento aumenta la resistencia de la cerámica porque disminuye la porosidad y proporciona una distribución más uniforme de los cristales en el seno de la matriz.

\section{Tecnología asistida por ordenador}

Hoy en día, la tecnología CAD-CAM (Computer Aid Design - Computer Aid Machining) nos permite confeccionar restauraciones cerámicas precisas de una forma rápida y cómoda. Todos estos sistemas controlados por ordenador constan de tres fases: digitalización, diseño y mecanizado. Gracias a la digitalización se registra tridimensionalmente la preparación dentaria. Esta exploración puede ser extraoral (a través de una sonda mecánica o un láser se escanea la superficie del troquel o del patrón) o intraoral (en la que una cámara capta directamente la imagen del tallado, sin necesidad de tomar impresiones). Estos datos se transfieren a un ordenador donde se realiza el diseño con un software especial. Concluido el diseño, el ordenador da las instrucciones a la unidad de fresado, que inicia de forma automática el mecanizado de la estructura cerámica. Los sistemas más representativos son Cerec ${ }^{\circledR}$ (Sirona), Procera ${ }^{\circledR}$ (Nobel Biocare), Lava ${ }^{\circledR}$ (3M Espe), DCS $®$ (DCS), Cercon $®$ (Dentsply), Everest $\circledast$ (Kavo), Hint-Els $\circledast$ (Hint-Els), etc. Actualmente, no existe suficiente evi- 
dencia científica para determinar cual es el mejor procedimiento. Sin embargo, en lo que si están de acuerdo la mayoría de los autores es que en el futuro, la tecnología CAD/CAM se impondrá a la técnica de confección manual.

Con las técnicas descritas se puede realizar el volumen completo de la restauración y luego proceder a su caracterización mediante maquillaje superficial; o se puede confeccionar la estructura interna y luego terminarla mediante la aplicación de capas de porcelana feldespática convencional. El maquillaje superficial se utiliza más en incrustaciones y carillas. Mientras que la estratificación de capas es el método ideal para coronas y puentes, ya que nos permite obtener mejores resultados estéticos porque el color se consigue desde las capas profundas.

\section{Criterios de selección}

Como hemos comprobado, en la actualidad disponemos de un amplio espectro de cerámicas con propiedades y aplicaciones muy diferentes en función de su composición química y proceso de síntesis. Por ello, a la hora de seleccionar el sistema cerámico más adecuado, resulta vital conocer el comportamiento de estos materiales analizando los requisitos básicos que se le pide a cualquier prótesis fija: resistencia a la fractura, precisión de ajuste marginal, estética y supervivencia clínica.

\section{Resistencia a la fractura}

Uno de los principales problemas que afecta la vida de las restauraciones es la fractura de la cerámica. En

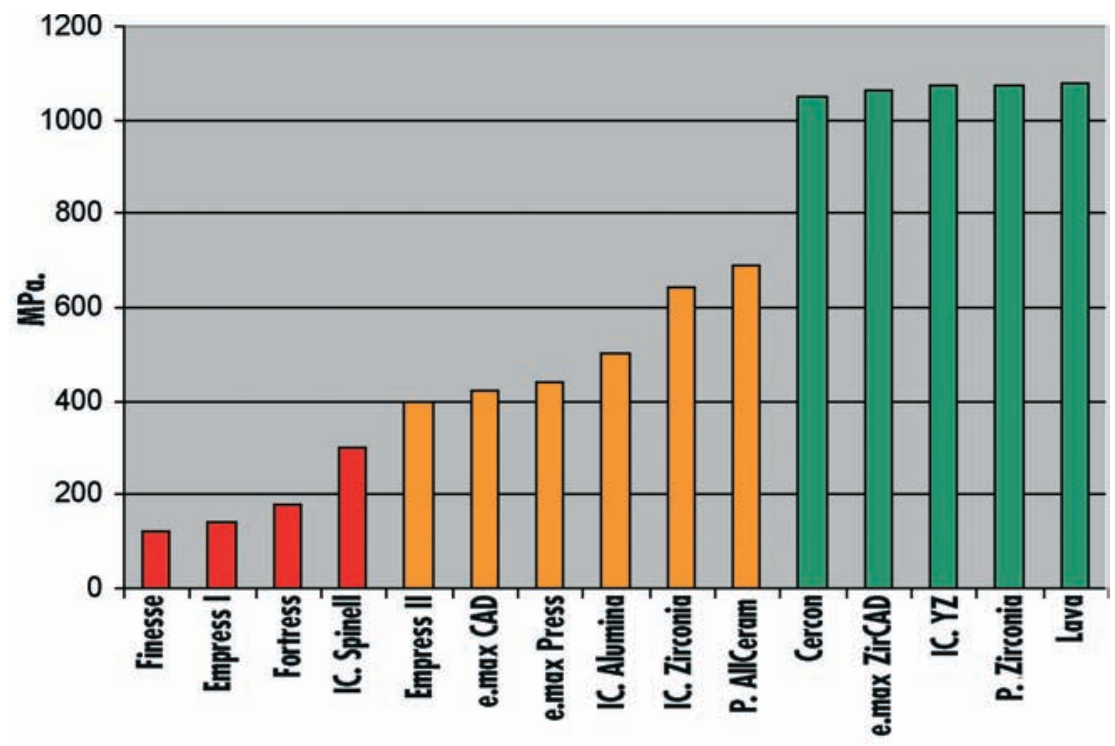

Figura 3. Resistencia a la fractura de distintos sistemas cerámicos (ISO 6872).

teoría, todos los sistemas actuales poseen una adecuada resistencia a la fractura porque todos superan el valor límite de $100 \mathrm{MPa}$, establecido por la norma ISO 6872. Pero la realidad es que existen diferencias considerables entre unos y otros (fig. 3). Por este motivo, creemos que es más correcto utilizar como punto de referencia la resistencia de las restauraciones metal-cerámica, que está comprendida entre los 400 y $600 \mathrm{MPa}^{5}$. De manera que podemos clasificar a las cerámicas sin metal en tres grupos:

- Baja resistencia (100-300 MPa): En el que se sitúan las porcelanas feldespáticas.

- Resistencia moderada (300-700 $\mathrm{MPa})$ : Representado fundamentalmente por las aluminosas, aunque también incluimos a IPS Empress II e IPS e.max Press/CAD (Ivoclar).

- Alta resistencia (por encima de 700 $\mathrm{MPa}$ ): En el que quedarían encuadradas todas las cerámicas circoniosas.
Esta clasificación tiene una gran importancia clínica, ya que nos permite delimitar las indicaciones de los distintos materiales cerámicos. Como ya señalamos, los sistemas circoniosos debido a sus elevados valores se han convertido en los candidatos idóneos para elaborar prótesis cerámica en zonas de alto compromiso mecánico. Sin embargo, no podemos olvidar que estos datos se refieren exclusivamente a las estructuras de circonia. En la práctica clínica, estas prótesis incorporan porcelana de recubrimiento, que presenta unas propiedades mecánicas distintas. En este sentido, varios autores han observado que las restauraciones circoniosas in vivo no son tan resistentes como predicen los trabajos in vitro ${ }^{6}$. Así, sundh \& cols. demostraron que el recubrimiento cerámico disminuía notablemente la tenacidad de la circonia, justo al contrario de lo que ocurre en los cerámicas feldespáticas y aluminosas?. Cuanto más frágil es el núcleo, mayor es el 
refuerzo que ejerce la porcelana de recubrimiento. A medida que se aumenta la tenacidad de la estructu$\mathrm{ra}$, se pierde el efecto de blindaje de la porcelana de recubrimiento. Por lo tanto, debemos de ser cautos a la hora de indicar estas restauraciones porque aunque su resistencia supere a la del resto de cerámicas, todavía queda mucho camino por recorrer antes de que estos sistemas estén en condiciones de sustituir a la técnica metal-cerámica en su empleo cotidiano.

No obstante, sabemos que la resistencia de una restauración también depende de una serie de factores clínicos como son: la preparación dentaria, el diseño de la estructura y el cementado. Si se manejan de forma adecuada, la probabilidad de fractura se reduce significativamente. Pero, no vamos a entrar en detalles porque se salen de los objetivos de este artículo.

\section{Precisión de ajuste margi- nal}

Para poder hablar de éxito en prótesis fija es imprescindible conseguir un buen sellado marginal. Las restauraciones indirectas, al confeccionarse fuera de boca y posteriormente fijarse a la preparación, generan una interfase, es decir, siempre existe un espacio real o virtual entre el diente y la prótesis. La misión del agente cementante es rellenar esta interfase para aumentar la retención entre ambos elementos y mantener su integridad. La adaptación marginal tiene una gran importancia clínica, ya que los desajustes a este nivel son los responsables de una serie de alteraciones que van a desembocar con el paso del tiempo en el fracaso del tra- tamiento. Por lo tanto, para garantizar la longevidad de una restauración es fundamental que la interfase preparación-prótesis sea mínima. Indudablemente, el ajuste perfecto es aquel en el que el margen de la restauración coincide con el ángulo cavosuperficial del diente. Pero como esta situación es difícil de alcanzar, siempre se acepta cierto grado de discrepancia.

Actualmente, no disponemos de un consenso sobre el tamaño de interfase aceptable desde el punto de vista clínico, ya que en la adaptación final de una prótesis fija influyen múltiples variables entre las que cabe señalar: la preparación dentaria, la técnica de confección de la restauración, la selección del agente cementante y la técnica de cementado. Al revisar la bibliografía observamos que hay un amplio intervalo de valores empíricos comprendido entre 5 y 200 $\mu \mathrm{m}$, hecho que pone de manifiesto la ausencia de un límite objetivo basado en la evidencia científica. Sin embargo, la mayoría de los autores admiten $120 \mu \mathrm{m}$ como el desajuste máximo tolerable. Teniendo en cuenta este dato podemos afirmar que los actuales sistemas cerámicos ofrecen unos ajustes marginales adecuados, siendo en muchos casos inferiores a los obtenidos con la metal-cerámica (40-70 $\mu \mathrm{m})^{8}$.

\section{Estética}

La estética es otro factor determinante en la elección de estos sistemas. En la clínica diaria, la mayoría de las situaciones las resolvemos con las técnicas ceramometálicas, y no cabe duda de que con estas restauraciones se consiguen unos resultados estéticos más que aceptables, pero nunca

\begin{tabular}{|ll|}
\hline \multicolumn{1}{|c|}{ Tabla l. Clasificación } \\
de los sistemas cerámicos \\
por sU grado de trans|ucidez \\
\hline Translúcidas & \multicolumn{1}{c|}{ opacas } \\
\hline Finesse & In-Ceram Alumina \\
Fortress & In-Ceram Zirconia \\
Optec-HSP & Procera AllCeram \\
IPS Empress I & Procera Zirconia \\
IPS Empress II & IPS e.max ZirCAD \\
IPS e.max CAD & Cercon \\
IPS e.max Press & DC-Zirkon \\
In-Ceram Spinell & Lava \\
& In-Ceram YZ \\
\hline
\end{tabular}

alcanzan la naturalidad de la prótesis cerámica. Esto se debe a que la cofia metálica impide el paso de la luz, reduciendo la profundidad del color. En cambio, la cerámica sin metal, al permitir la transmisión de la luz a través del cuerpo del diente, consigue mayor mimetismo. Sin embargo, a pesar de que las restauraciones totalmente cerámicas son siempre más estéticas que las ceramometálicas, existen diferencias entre ellas. Estas diferencias radican fundamentalmente en el grado de translucidez de estos materiales. Así, podemos clasificar a los sistemas cerámicos en dos grupos en función de su comportamiento estético: translúcidos y opacos (tabla 1).

En este punto es importante recordar que la matriz vítrea es la responsable de la translucidez de la porcelana. Por lo tanto, en el primer grupo se encuentran aquellas cerámicas que tienen una mayor fase vítrea, es decir, las feldespáticas. También incluimos en este apartado 


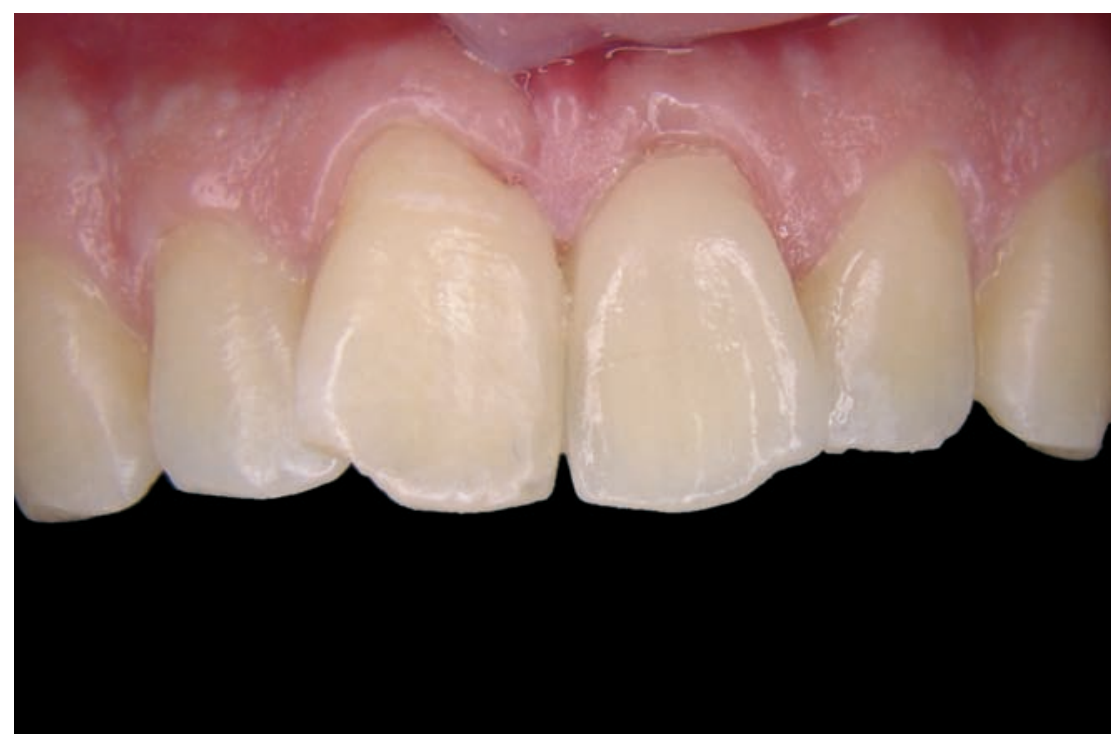

Figura 4. Corona cerámica Procera ${ }^{\circledR}$ AllCeram en incisivo central superior izquierdo.

a In-Ceram Spinell porque, a pesar de que se trata de una porcelana aluminosa, su núcleo es bastante translúcido debido a que la espinela es un cristal con unas buenas propiedades ópticas. Dentro de este grupo destaca IPS e.max Press, ya esta cerámica dispone de dos grados de opacidad, uno bajo para dientes vitales y otro alto para enmascarar sustratos oscuros. En el segundo grupo, situamos a las cerámicas aluminosas y circoniosas ya que apenas tienen fase vítrea y por lo tanto, son menos transparentes. Dentro de este grupo destacan los sistemas circoniosos Lava e InCeram YZ porque su translucidez es variable. Su grado de translucidez se puede controlar mediante dos factores: el grosor de la estructura porque lógicamente a mayor espesor, más opacidad y otro, es el color de la estructura, ya que estos núcleos se pueden colorear en siete tonos. Este aspecto es importante tenerlo en cuenta a la hora de seleccionar el sis- tema cerámico, ya que en función del color de sustrato elegiremos una cerámica translúcida u opaca.

Pero al hablar de estética no solo es importante contar con un material que cumpla los requisitos adecuados de color y translucidez sino que hay que considerar otras variables que a nuestro juicio tienen más trascendencia en el resultado final. Así, para conseguir un aspecto estético aceptable es fundamental la obtención de formas anatómicas naturales (fig. 4). La labor del ceramista en este aspecto es vital, puesto que debe ser un perfecto copista de la anatomía dentaria. La simetría y proporcionalidad son también factores condicionantes de la estética, ya que dientes asimétricos o de proporciones exageradas respecto a los dientes remanentes van a alterar la armonía y el equilibrio global de la sonrisa?.

\section{Supervivencia clínica}

La valoración clínica es fundamental en la evaluación de un sistema cerámico. Sabemos que en la práctica real interactúan una serie de variables (como son las características oclusales, presencia de hábitos parafuncionales, grado de higiene, etc.) prácticamente impredecibles en las investigaciones in vitro, y que sin embargo son absolutamente primordiales en la vida de las restauraciones. Por ello, es fundamental revisar siempre los estudios clínicos. Solamente, de esta manera podremos tomar una decisión objetiva basada en la evidencia científica.

Al analizar las investigaciones sobre incrustaciones cerámicas ${ }^{10-13}$, observamos que los resultados obtenidos con los sistemas feldespáticos Empress y Cerec-Vitablocs son los mejores, ya que tienen una supervivencia clínica a medio plazo superior al 90\%. Sin

\begin{tabular}{|c|c|c|c|}
\hline \multicolumn{4}{|c|}{ Tabla e. Estudios clinnicos de incrusłaciones } \\
\hline $\begin{array}{l}\text { Material } \\
\text { restaurador }\end{array}$ & $\begin{array}{l}\text { Período de } \\
\text { observación }\end{array}$ & $\begin{array}{c}\text { Tasa de } \\
\text { supervivencia }\end{array}$ & Investigadores \\
\hline IPS e.max Press & 2 años & $100 \%$ & Guess \& cols, 2006 \\
\hline IPS Empress I & 7 años & $91 \%$ & El-Mowafy \& Brochu, 2002 \\
\hline Mirage & 9 años & $84 \%$ & Schulz \& cols, 2003 \\
\hline Cerec - Vitablocs Mark & 10 años & $90 \%$ & Fasbinder, 2006 \\
\hline Oro & 25 años & $94,9 \%$ & Donovan \& cols, 2004 \\
\hline
\end{tabular}




\begin{tabular}{|c|c|c|c|}
\hline \multicolumn{4}{|c|}{ Tabla 3. Estudios clínicos de carillas } \\
\hline $\begin{array}{l}\text { Material } \\
\text { restaurador }\end{array}$ & $\begin{array}{l}\text { Período de } \\
\text { observación }\end{array}$ & $\begin{array}{c}\text { Tasa de } \\
\text { supervivencia }\end{array}$ & Investigadores \\
\hline Ceramco & 5 años & $98,4 \%$ & Aristidis \& Dimitra, 2002 \\
\hline Cerec - Vitablocs Mark & rk 9 años & $94 \%$ & Wiedhahn \& cols, 2005 \\
\hline Optec-HSP & 10 años & $91 \%$ & Dumfahrt \& Schaffer, 2000 \\
\hline IPS Empress I & 12 años & $94,4 \%$ & Fradeani \& cols, 2005 \\
\hline Resina compuesta & 2,5 años & $80 \%$ & Meijering \& cols, 1998 \\
\hline
\end{tabular}

\begin{tabular}{|lccc|}
\hline \multicolumn{4}{|c|}{ Tabla 4. Estudios clínic0S de CO「OחaS } \\
\hline Material & $\begin{array}{c}\text { Período de } \\
\text { observación }\end{array}$ & $\begin{array}{c}\text { Tasa de } \\
\text { supervivencia }\end{array}$ & Investigadores \\
\hline restaurador & 4 años & $100 \%$ & Haselton \& cols, 2000 \\
In Ceram Alumina & 5 años & $97,5 \%$ & Fradeani \& cols, 2002 \\
In Ceram Spinell & 5 años & $96,7 \%$ & Fradeani \& cols, 2005 \\
Procera All Ceram & 5 años & $100 \%$ & Marquardt \& Strub, 2006 \\
IPS Empress II & 10 años & $94 \%$ & Walton, 1999 \\
Metal-cerámica & & & \\
\hline
\end{tabular}

\begin{tabular}{|lccc|}
\hline \multicolumn{4}{|c|}{ Tabla 5. Estudios clínicos de puentes } \\
\hline Material & $\begin{array}{c}\text { Período de } \\
\text { observación }\end{array}$ & $\begin{array}{c}\text { Tasa de } \\
\text { supervivencia }\end{array}$ & Investigadores \\
\hline restaurador & 3 años & $100 \%$ & Raigrodski \& cols, 2006 \\
\hline Lava & 3 años & $94,5 \%$ & Suárez \& cols, 2004 \\
In Ceram Zirconia & 5 años & $93 \%$ & Olsson \& cols, 2003 \\
In Ceram Alumina & 5 años & $70 \%$ & Marquardt \& Strub, 2006 \\
IPS Empress II & 10 años & $87 \%$ & Walton, 2002 \\
Metal-cerámica & & \multicolumn{3}{c}{} \\
\hline
\end{tabular}

\begin{tabular}{|lccc|}
\hline \multicolumn{4}{|c|}{ Tabla 6. Estudios clinicos de pilares implantosoporlados } \\
\hline $\begin{array}{l}\text { Material } \\
\text { restaurador }\end{array}$ & $\begin{array}{c}\text { Período de } \\
\text { observación }\end{array}$ & $\begin{array}{c}\text { Tasa de } \\
\text { supervivencia }\end{array}$ & Investigadores \\
\hline Alúmina & 5 años & $94,7 \%$ & Andersson \& cols, 2003 \\
\hline Circonia & 4 años & $100 \%$ & Glauser \& cols, 2004 \\
\hline Titanio & 5 años & $100 \%$ & Andersson \& cols, 2003 \\
\hline
\end{tabular}

embargo, no alcanzan el éxito de las incrustaciones de oro $^{14}$ (tabla 2). Las carillas confeccionadas con cerámicas feldespáticas como Optec o IPS Em- press presentan unas tasas de supervivencia en torno al 90-95\%, demostrando un comportamiento clínico superior al de las carillas directas de resina ${ }^{15-}$
${ }_{19}$ (tabla 3). Respecto a las coronas, InCeram, Procera AllCeram e IPS Empress II son los únicos sistemas contrastados en la actualidad ${ }^{20-23}$. Sus resultados a medio plazo son excelentes e incluso comparables a los obtenidos con coronas metal-cerámica ${ }^{24}$. No obstante, deben de realizarse más estudios para evaluar el rendimiento clínico de estos sistemas a largo plazo y aclarar si la tasa de supervivencia es menor en los sectores posteriores en comparación con la región anterior (tabla 4).

Los estudios clínicos sobre puentes cerámicos son muy escasos y de corta duración ${ }^{23,25-27}$. A pesar de ello, los resultados más esperanzadores se han obtenido con los sistemas de alto contenido en circonia. Estas cifras confirman que el oxido de circonio debido a sus excelentes propiedades mecánicas es el material ideal para realizar puentes cerámicos (tabla 5). Sin embargo, con estos datos no se puede todavía recomendar su uso clínico a gran escala sin hacer reservas. Estos resultados deben ser respaldados por nuevos estudios longitudinales. Lo mismo sucede con los pilares implantosoportados (tabla 6), la circonia presenta una tasa de supervivencia superior a la alúmina y equiparable al titanio ${ }^{29,30}$. No obstante, la escasa evidencia científica disponible sobre este tema obliga a ser prudente.

\section{Conclusiones}

Una vez que hemos analizado los distintos criterios de selección, vamos a establecer las indicaciones de estos materiales. En principio, para plantearnos el uso de los sistemas totalmen- 
te cerámicos es necesario que se cumplan dos premisas:

- Que los requerimientos estéticos del caso sean máximos.

- Que haya un adecuado apoyo y experiencia del laboratorio con la cerámica seleccionada. Desde el punto de vista técnico, se requiere un ceramista que domine perfectamente el proceso de elaboración para lograr los resultados deseados. En algunos sistemas, la técnica es muy compleja porque se maneja aparatología específica, que requiere una gran inversión de tiempo y de dinero por parte del laboratorio.

Como contraindicaciones formales, solamente tenemos dos: presencia de hábitos parafuncionales y que el espacio protésico sea crítico como ocurre en mordidas cruzadas y sobremordidas profundas. Si se tienen en cuenta estas consideraciones que acabamos de exponer, podremos trabajar con estos sistemas de una forma segura. Pero cuando estas condiciones no se cumplen o el paciente exige garantías en la duración de la restauración, el material de elección es la metal-cerámica. Sólo se deben usar estas técnicas cuando el clínico este seguro de que el paciente está dispuesto a aceptar cierto riesgo en beneficio de una mayor estética.

Por último, a modo de resumen, vamos a exponer unas pautas clínicas que nos orienten en la elección de la cerámica en función del caso:

- Para realizar incrustaciones cerámicas, elegiremos las porcelanas feldespáticas ya que son las únicas que nos permiten realizar restauraciones conservadoras manteniendo el binomio estética-resistencia.

- Por los mismos motivos, emplearemos las cerámicas feldespáticas para hacer carillas. La única consideración es que cuando estemos ante un sustrato oscuro deberemos usar sistemas que nos permitan controlar el grado de translucidez.

- A la hora de elegir el sistema cerámico para confeccionar coronas en el sector anterior habrá que evaluar el color del sustrato:

- En sustratos claros, preferimos cerámicas feldespáticas porque al ser más translúcidas nos permiten un mayor mimetismo con los dientes naturales.

- En sustratos oscuros, es más adecuado emplear cerámicas aluminosas o circoniosas con cofias opacas que impidan que se transparente el color subyacente.

- En las coronas posteriores, el criterio que prima en la elección del material es la resistencia a la fractura. Por eso, elegiremos entre las cerámicas aluminosas o circoniosas, ya que sus propiedades mecánicas cumplen sobradamente con los requerimientos de estas restauraciones.

- Para puentes cerámicos, tanto del sector anterior como posterior, optaremos siempre por un sistema circonioso teniendo en cuenta las consideraciones que hemos comentado anteriormente, ya que sus resultados todavía no son equiparables a los de las restauraciones ceramometálicas.

- Lo mismo ocurre con los pilares implantosoportados cerámicos. En principio como primera opción usaremos los pilares circoniosos por su mayor tenacidad. Pero tampoco podemos olvidar que su resistencia es inferior a la de los pilares de titanio y que no disponemos de estudios clínicos a largo plazo.

Como hemos visto, todavía no existe el material cerámico ideal que cumpla a la perfección todos los requisitos. Lo ideal sería lograr la regeneración tisular empleando cerámicas biodegradables, que solamente permaneciesen en el organismo mientras fuese necesaria su función y desapareciesen a medida que los tejidos se fuesen regenerando. A pesar de que ya se ha conseguido experimentalmente crear dentina, los resultados de estos trabajos todavía están muy lejos de la clínica diaria. 


\section{Bibliografía recomendada}

Para profundizar en la lectura de este tema, el/los autor/es considera/an interesantes los artículos que aparecen señalados del siguiente modo: *de interés * ${ }^{*}$ de especial interés.

1. Vega JM. Porcelanas y cerámicas actuales. RCOE 1999;4:41-54.

2. Fons-Font A, Solá-Ruiz MF, Martínez-González A, Casas-Terrón J. Clasificación actual de las cerámicas dentales. RCOE 2001;6:645-56.

3. Álvarez-Fernández MA, Peña-López JM, González-González IR, Olay-García MS. Características generales y propiedades de las cerámicas sin metal. RCOE 2003;8:525-46.

4. de Aza AH, Chevalier J, Fantozzi G, Schehl M, Torrecillas R. Slow-crack-growth behavior of zirconia toughened alumina ceramic processed by different methods. J Am Ceram Soc 2003;86:115-120.

5**. Mallat E, Mallat E. Porcelana. En: Mallat E, Mallat E. Fundamentos de la estética bucal en el grupo anterior. Barcelona: Quintessence, 2001: 155-186.

Capítulo de referencia para el conocimiento de las cerámicas dentales, sus técnicas de confección y aplicaciones clínicas.

$6^{* *}$. Denry I, Kelly JR. State of the art of zirconia for dental applications. Dent Mater. En prensa 2007.

Excelente revisión crítica de las cerámicas de alto contenido en circonia, donde se analizan los problemas clínicos que presentan estos materiales.

7. Sundh A, Molin M, Sjögren G. Fracture resistance of yttrium oxide partially-stabilized zirconia all-ceramic bridges after veneering and mechanical fatigue testing. Dent Mater 2005;21:476-82.

8. Martínez Rus F. Estudio experimental del ajuste marginal en coronas cerámicas de alto contenido en circonia. Tesis Doctoral. Universidad Complutense de Madrid. Facultad de Odontología. Madrid 2005.

9. Suárez MJ, López JF, Salido MP, Serrano B. Coronas de recubrimiento total cerámicas. Criterios de selección. Rev Euro Odontoestomatol 1999;11:249-58.

10. Guess PC, Stappert CF, Strub JR. Preliminary clinical results of a prospective study of IPS e.max Press and Cerec ProCAD partial cove- rage crowns. Schweiz Monatsschr Zahnmed 2006;11:493-500.

11. El-Mowafy O, Brochu JF. Longevity and clinical performance of IPS-Empress ceramic restorations. A literature review. J Can Dent Assoc 2002;68:233-7.

12. Schulz P, Johansson A, Arvidson K. A retrospective study of Mirage ceramic inlays over up to 9 years. Int J Prosthodont 2003;16:510-4.

13. Fasbinder DJ. Clinical performance of chairside CAD/CAM restorations. J Am Dent Assoc 2006;137 Suppl:22-31.

14*. Donovan T, Simonsen RJ, Guertin G, Tucker RV. Retrospective clinical evaluation of 1,314 cast gold restorations in service from 1 to 52 years. J Esthet Restor Dent 2004;16:194-204. En este artículo se demuestra que las restauraciones coladas de oro tienen un excelente pronóstico a largo plazo. Los resultados obtenidos son inalcanzables para cualquier material restaurador actual.

15. Aristidis GA, Dimitra B. Five-year clinical performance of porcelain laminate veneers. Quintessence Int 2002;33:185-9.

16. Wiedhahn K, Kerschbaum T, Fasbinder DF. Clinical long-term results with 617 Cerec veneers: a nine-year report. Int J Comput Dent 2005;8:233-46.

17. Dumfahrt H, Schaffer H. Porcelain laminate veneers. A retrospective evaluation after 1 to 10 years of service: Part II-Clinical results. Int J Prosthodont 2000;13:9-18.

18. Fradeani M, Redemagni M, Corrado M. Porcelain laminate veneers: 6- to 12-year clinical evaluation--a retrospective study. Int J Periodontics Restorative Dent 2005;25:9-17.

19. Meijering AC, Creugers NH, Roeters FJ, Mulder J. Survival of three types of veneer restorations in a clinical trial: a 2.5 year interim evaluation. J Dent 1998;26:563-8.

20. Haselton DR, Diaz-Arnold AM, Hillis SL. Clinical assessment of high-strength all-ceramic crowns. J Prosthet Dent 2000;83:396-401.

21. Fradeani M, Aquilano A, Corrado M. Clinical experience with In-Ceram Spinell crowns: 5year follow-up. Int $\mathrm{J}$ Periodontics Restorative Dent 2002;22:525-33.

22*. Fradeani M, D'Amelio M, Redemagni M, Corrado M. Five-year follow-up with Procera all-ceramic crowns. Quintessence Int 2005;36: 105-13.

Estudio clínico que avala el buen comportamiento de las coronas cerámicas Procera AllCeram.

23. Marquardt P, Strub JR. Survival rates of IPS empress 2 all-ceramic crowns and fixed partial dentures: results of a 5-year prospective clinical study. Quintessence Int 2006;37:253-9.

24. Walton TR. A 10-year longitudinal study of fixed prosthodontics: clinical characteristics and outcome of single-unit metal-ceramic crowns. Int J Prosthodont 1999;12:519-26.

25. Raigrodski AJ, Chiche GJ, Potiket $\mathrm{N}$ y et al. The efficacy of posterior three-unit zirconiumoxide-based ceramic fixed partial dental prostheses: a prospective clinical pilot study. J Prosthet Dent 2006;96:237-44.

26. Suarez MJ, Lozano JF, Paz Salido M, Martinez F. Three-year clinical evaluation of In-Ceram Zirconia posterior FPDs. Int J Prosthodont 2004; 17:35-8.

27. Olsson KG, Furst B, Andersson B, Carlsson GE. A long-term retrospective and clinical followup study of In-Ceram Alumina FPDs. Int J Prosthodont 2003;16:150-6.

28. Walton TR. An up to 15-year longitudinal study of 515 metal-ceramic FPDs: Part 1. Outcome. Int J Prosthodont 2002;15:439-45.

29. Andersson B, Glauser R, Maglione M, Taylor A. Ceramic implant abutments for short-span FPDs: a prospective 5-year multicenter study. Int J Prosthodont 2003;16:640-6.

30. Glauser R, Sailer I, Wohlwend A, Studer S, Schibli M, Scharer P. Experimental zirconia abutments for implant-supported singletooth restorations in esthetically demanding regions: 4-year results of a prospective clinical study. Int J Prosthodont 2004;17:285-90. 